Stephen S. Harlan

Appendix 1.

\title{
Analytical Methods and Results
}

Baddeleyite, and in some cases zircon, were separated by crushing, grinding, and concentration by Wilfley table and heavy liquids. The baddeleyite consisted of tiny ( $200 \mathrm{Pm})$ red-brown, translucent, striated and bladed crystals and crystal fragments. In general, the baddeleyite grain size in dike samples was considerably smaller $(<60 \mathrm{Pm})$ than in gabbro sheets, resulting in low crystal yields and precluding multiple baddeleyite U-Pb analyses in some instances. The zircons, obtained from the southern Tobacco Root dikes consisted of small euhedral grains that were commonly cracked. They provided strongly discordant results and are not considered further. Isotopic compositions for $\mathrm{U}$ and $\mathrm{Pb}$ were obtained through thermal-ionization mass spectrometry using procedures described in French et al. (2002) for the analyses done at the University of Alberta laboratory and in Harlan et al. (2003) for those done at the U.S. Geological Survey laboratory in Denver. All age calculations were determined by using ISOPLOT/Ex (Ludwig, 2003). Except where otherwise indicated, uncertainties in isotopic ages are reported at the $95 \%$ confidence level.

TABLE DR1. U-Pb BADDELEYITE RESULTS FOR GUNBARREL IGNEOUS EVENTS

\begin{tabular}{|c|c|c|c|c|c|c|c|c|c|c|c|c|}
\hline $\begin{array}{c}\text { Sample } \\
\text { No. }\end{array}$ & $\begin{array}{c}\text { Mass } \\
(\mathrm{Pg})\end{array}$ & $\underset{(\mathrm{ppm})}{U}$ & $\begin{array}{l}\mathrm{Th}^{*} \\
(\mathrm{ppm})\end{array}$ & $\begin{array}{c}\mathrm{Pb} \\
(\mathrm{ppm})\end{array}$ & Th/U & $\begin{array}{l}\mathrm{Pb}^{\dagger} \\
(\mathrm{pg})\end{array}$ & ${ }^{206} \mathrm{~Pb} /{ }^{204} \mathrm{~Pb}$ & ${ }^{206} \mathrm{~Pb} /{ }^{238} \mathrm{U}$ & ${ }^{207} \mathrm{~Pb} /{ }^{235} \mathrm{U}$ & ${ }^{207} \mathrm{~Pb} /{ }^{206} \mathrm{~Pb}$ & $\begin{array}{c}{ }^{207} \mathrm{~Pb} /{ }^{206} \mathrm{~Pb} \\
\text { Model age } \\
\text { (Ma) }\end{array}$ & $\begin{array}{c}\begin{array}{c}\text { Discor- } \\
\text { dance }\end{array} \\
(\%)\end{array}$ \\
\hline \multicolumn{13}{|c|}{ HY8A-91: Gunbarrel gabbro, Canada $\left(65.6611^{\circ} \mathrm{N}, 118.1444^{\circ} \mathrm{W}\right)$} \\
\hline 6 & 10 & 479 & 31 & 57 & 0.06 & 13 & 2894 & $0.12591 \pm 14$ & $1.1306 \pm 16$ & $0.06512 \pm 5$ & $778.3 \pm 1.6$ & 1.9 \\
\hline 7 & 11 & 461 & 28 & 54 & 0.06 & 6 & 6322 & $0.12563 \pm 15$ & $1.1293 \pm 16$ & $0.06519 \pm 5$ & $780.6 \pm 1.7$ & 2.4 \\
\hline \multicolumn{13}{|c|}{ FA149-62: Calder gabbro sheet, Canada $\left(65.55^{\circ} \mathrm{N}, 117.22^{\circ} \mathrm{W}\right)$} \\
\hline 8 & 42 & 237 & 14 & 28 & 0.06 & 14 & 5834 & $0.12696 \pm 17$ & $1.1407 \pm 17$ & $0.06516 \pm 5$ & $779.7 \pm 1.5$ & 1.3 \\
\hline 9 & 60 & 134 & 8 & 16 & 0.06 & 25 & 2539 & $0.12563 \pm 17$ & $1.1282 \pm 17$ & $0.06513 \pm 6$ & $778.5 \pm 2.0$ & 2.1 \\
\hline 10 & 35 & 312 & 27 & 38 & 0.09 & 15 & 5684 & $0.12719 \pm 14$ & $1.1429 \pm 14$ & $0.06517 \pm 5$ & $779.9 \pm 1.5$ & 1.1 \\
\hline \multicolumn{13}{|c|}{ GFA92-567: Faber Lake gabbro sheet $\left(64.12^{\circ} \mathrm{N}, 116.67^{\circ} \mathrm{W}\right)$} \\
\hline 1 & 42 & 1158 & 81 & 137 & 0.07 & 19 & 20,065 & $0.12615 \pm 17$ & $1.1332 \pm 17$ & $0.06515 \pm 3$ & $779.2 \pm 1.0$ & 1.8 \\
\hline 2 & 42 & 1323 & 56 & 157 & 0.04 & 37 & 12,083 & $0.12717 \pm 13$ & $1.1428 \pm 14$ & $0.06517 \pm 3$ & $779.9 \pm 1.0$ & 1.1 \\
\hline
\end{tabular}

NW13-2: Concajou Canyon gabbro sill, Canada $\left(64.17^{\circ} \mathrm{N}, 127.97^{\circ} \mathrm{W}\right)$ 


\begin{tabular}{|c|c|c|c|c|c|c|c|c|c|c|c|c|}
\hline 3 & 18 & 514 & 27 & 62 & 0.05 & 25 & 2990 & $0.12618 \pm 19$ & $1.1341 \pm 17$ & $0.06519 \pm 7$ & $780.4 \pm 2.2$ & 2.0 \\
\hline 4 & 15 & 62 & 39 & 72 & 0.06 & 16 & 4489 & $0.12631 \pm 14$ & $1.1346 \pm 15$ & $0.06515 \pm 4$ & $779.2 \pm 1.4$ & 1.7 \\
\hline \multicolumn{13}{|c|}{ 91T2-2: Muncho Lake diabase dike, Canada $\left(58.78^{\circ} \mathrm{N}, 125.67^{\circ} \mathrm{W}\right)$} \\
\hline 5 & 4 & 786 & 56 & 93 & 0.07 & 5 & 5168 & $0.12598 \pm 15$ & $1.1306 \pm 17$ & $0.06509 \pm 5$ & $777.3 \pm 1.5$ & 1.7 \\
\hline \multicolumn{13}{|c|}{ 93T258-1: Christmas Lake diabase dike, Wyoming $\left(44.98^{\circ} \mathrm{N}, 109.43^{\circ} \mathrm{W}\right)$} \\
\hline 11 & 4 & 947 & 90 & 114 & 0.10 & 7 & 4508 & $0.12673 \pm 13$ & $1.1385 \pm 15$ & $0.06516 \pm 5$ & $779.5 \pm 1.5$ & 1.4 \\
\hline 12 & 2 & 557 & 54 & 66 & 0.10 & 3 & 2502 & $0.12384 \pm 25$ & $1.1069 \pm 50$ & $0.06483 \pm 26$ & $768.7 \pm 8.4$ & 2.2 \\
\hline \multicolumn{13}{|c|}{ TR-7: Southern Tobacco Root Mountains dike, Montana $\left(45.4156^{\circ} \mathrm{N}, 112.1327^{\circ} \mathrm{W}\right)$} \\
\hline $5(43)$ & n.d. (60 gr) & - & - & - & - & - & 2455 & $0.12689 \pm 19$ & $1.1413 \pm 29$ & $0.06524 \pm 13$ & $782 \pm 4$ & 1.9 \\
\hline $6(43)$ & n.d. & - & - & - & - & - & 1402 & $0.11759 \pm 12$ & $1.0559 \pm 70$ & $0.06512 \pm 40$ & $778 \pm 13$ & 10.7 \\
\hline \multicolumn{13}{|c|}{ TR-19: Southern Tobacco Root Mountains dike, Montana $\left(45.4156^{\circ} \mathrm{N}, 112.1327^{\circ} \mathrm{W}\right)$} \\
\hline $5(47)$ & $\begin{array}{l}\text { n.d. }(37 \text { gr }) \\
\text { n.d. }\end{array}$ & - & - & - & - & - & $\begin{array}{l}1990 \\
3011\end{array}$ & $\begin{array}{l}0.12563 \pm 48 \\
0.11525 \pm 111\end{array}$ & $\begin{array}{l}1.1283 \pm 43 \\
1.0331 \pm 10\end{array}$ & $\begin{array}{l}0.06514 \pm 23 \\
0.06502 \pm 18\end{array}$ & $\begin{array}{l}779 \pm 7 \\
775 \pm 6\end{array}$ & $\begin{array}{c}3.3 \\
13.0\end{array}$ \\
\hline
\end{tabular}

Note: Atomic ratios are corrected for fractionation (0.1\%/amu), blank ( $2 \mathrm{pg} \mathrm{Pb} ; 0.5 \mathrm{pg} \mathrm{U}$ ), spike and common Pb (depleted mantle). All errors given in this table are $1 \mathrm{~V}$ uncertainties and refer to the least-significant digit(s); n.d. indicates masses were not determined; 60 and 37 gr refer to the number of individual grains analyzed, where mass of the sample is not reported. For the samples from the U.S. Geological Survey laboratory where individual sample masses were not determined, absolute values for $\mathrm{U}$, Th, and $\mathrm{Pb}$ concentrations are not reported.

${ }^{*}$ Th concentration estimated from abundance of ${ }^{208} \mathrm{~Pb}$ and corresponding ${ }^{207} \mathrm{~Pb} /{ }^{206} \mathrm{~Pb}$ ages.

Total amount of common $\mathrm{Pb}$ in picograms measured in the analysis.

${ }^{206} \mathrm{~Pb} /{ }^{204} \mathrm{~Pb}$ ratios are corrected for fractionation and spike only.

\section{References:}

French, J.E., Heaman, L.M., and Chacko, T., 2002, Feasibility of chemical U-Th-total Pb baddeleyite dating by electron microprobe: Chemical Geology, v. 188, p. 85-104.

Harlan, S.S., Geissman, J.W., and Premo, W.R., 2003, Paleomagnetism and geochronology of an Early Proterozoic quartz diorite in the southern Wind River Range, Wyoming, USA: Tectonophysics, v. 362, p. 105-122.

Ludwig, K.R., 2003, ISOPLOT/Ex, A geochronological toolkit for Microsoft Excel: Berkeley Geochronology Center Special Publication 4, $70 \mathrm{p}$. 